\title{
Research on Pre-coding Technology Analysis of LTE Systems
}

\author{
Zhiyong Ren
}

\author{
School of Communication Engineering, \\ ChongQing College of Electronic Engineering, ChongQing 401331, China
}

E-mail: renzhiyongtxxy@sina.com

\section{Keywords: LTE, Pre-coding Technology, Program Design}

\begin{abstract}
As the demand for wireless communications become more sophisticated, the wireless communication system also received an unprecedented flourish. LTE as the evolution of 3G systems, which greatly enhance the spectrum utilization system, it is possible to obtain higher than traditional wireless communication system throughput. In order to fully utilize LTE technology brings the system upgrade pre-coding technology, at the transmitter to send data preprocessing information based on the channel, so as to simplify the complexity of the receiver or the purpose of improving system performance. In order to meet the needs of LTE systems in high-rate and high capacity aspects, LTE system supports multiple input and output technology downstream applications, including spatial multiplexing, beamforming and pre-coding technology. LTE system pre-coding technology will help to assess the performance of LTE systems, and promote its practical application. The pre-coding technology would facilitate a more standardized process, and enhance the right to speak in the international field of standardization.
\end{abstract}

\section{Theoretical Introduction of LTE Technology}

LTE (Long Term Evolution) project is an evolution of 3G, LTE is not widespread misunderstanding of $4 \mathrm{G}$ technology, but a transition between $3 \mathrm{G}$ and $4 \mathrm{G}$ technology, is the world standard of 3.9G, it improves and enhances the 3G air access technology, the use of orthogonal frequency division multiplexing technology as the sole criterion for its wireless network evolution. LTE system improves cell edge user performance, and can improve cell capacity and reducing system latency. In this scenario, the role of the pre-coding technique is more important.

Compared with 3G, LTE has the following technical characteristics: improve communication speed downlink peak rate of $100 \mathrm{Mbps}$, the behavior of 50Mbps. Improve spectral efficiency, downlink 5 (bit/s) / Hz, 3-4 times the R6 version; uplink 2.5 (bit/s) / Hz, 2-3 times the R6 version. In the packet domain services as the main target, the overall architecture of the system will be based on packet switching. Reduce wireless network delay: sub-frame length is set to $0.5 \mathrm{~ms}$ and $0.675 \mathrm{~ms}$, and solve the problem of backwards compatibility and reduces network latency ${ }^{[1]}$.

LTE system requirements can be achieved at a bandwidth of up to $20 \mathrm{MHz} 100 \mathrm{Mbit} / \mathrm{s}$ downlink peak rate, so LTE wireless access technology with a new technology and MIMO OFDM technology. Because of its openness, it was supported by the vast majority of manufacturers to determine the LTE standard authoritative position in the industry. Some countries and regions have started commercial operation LTE systems attempt. In the actual system, due to the limited feedback channel capacity, using a codebook based pre-coding technology limited feedback. For a limited feedback precoding, the most important issue is the design of the code and the code word selection algorithm.

\section{The Basic Theory of Pre-Coding}

Pre-coding technology is known channel state information (Channel States Information, CSI) in the case, the sender use CSI transmission signal preprocessing operation, thereby further improving the throughput of the user and the system. Using signal processing according to different methods, it can be pre-coded into linear precoding and nonlinear precoding ${ }^{[2]}$. 
Linear precoding division position can be obtained according to the pre-coding matrix: non-codebook based precoding method and precoding codebook mode. In a frequency division duplex system, the priori information channel by channel MIMO measurements obtained and passed through a feedback channel to the sender. In time division duplex (Time Division Duplex, TDD) system, since uplink and downlink channels have reciprocity, downlink MIMO precoding channel information can be obtained by an uplink required channel measurement, i.e., achieve a non-feedback mode codebook.

LTE uses the flat network architecture, using a single-layer structure formed by the eNodeB. This structure facilitates simplified network and reduce the delay to achieve a low-latency, low complexity and low cost requirements. Compared with the conventional 3GPP access network, LTE radio network controller reduces (Radio Network Controller, RNC) node. Nominally LTE is the evolution of 3G, but in fact it made the whole architecture of 3G revolutionary change, and gradually close to the typical IP broadband network structure. LTE access network is mainly composed of two parts eNodeB and the access gateway. Access Gateway is a boundary node, if it is considered part of the core network, the access network is mainly composed of a eNodeB layer. In addition to the original eNodeB functionality, LTE network architecture also completed most of the functionality of the original radio network controller, including the physical layer, MAC (Media Access Control MAC) layer, RRC (Radio Resource Control RRC), scheduling, access control, bearer control, access to mobility management, using direct interconnection between eNode grids, which is a major modification of the original structure ${ }^{[3]}$. Fig.1 shows the LTE network architecture.

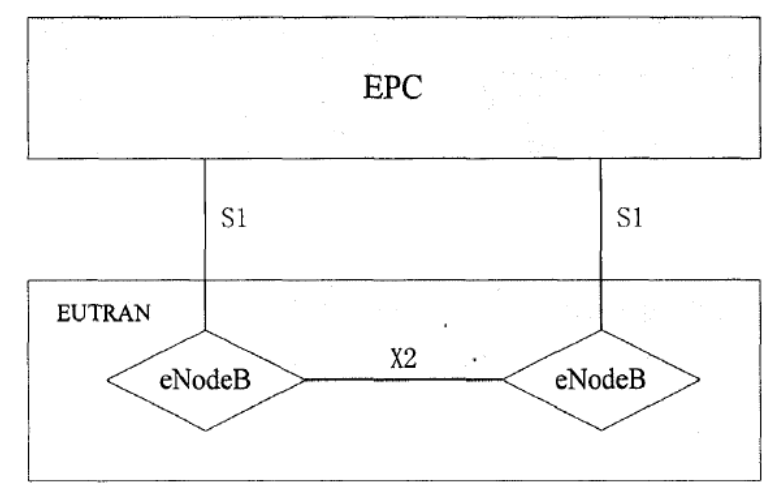

Fig. 1.The LTE network architecture

\section{The Design of LTE Precoding}

LTE candidate pre-coding techniques can be divided into two categories: non-codebook based pre-coding technology and codebook based pre-coding technology. Both operational processes are completely different. For precoding method based on a non-codebook precoding matrix is calculated by the sender. The sender sends the received pilot signal to estimate the channel state information, and then pre-coding matrix calculations. The sender is calculated after a pilot signal to send data and dedicated pilot signal pre-coding operation together. In the codebook-based precoding technique, the precoding matrix is calculated by the receiver. The receiver estimates the channel state information based on the current downlink pilot channel signal information, according to the channel information matrix selected from a codebook as the pre-coding matrix, then the matrix in the codebook index of the uplink channel feedback to the sender.

LTE supports two different pre-encoding: non-codebook based pre-coding and coding based on a pre-codebook. For non-codebook precoding method, it requires the receiving end in some way to channel information back to the transmitter, such as the use of symmetry TDD channel, direct feedback channel to quantify and check points or feedback. After the transmitter channel information can be directly obtained in accordance with the guidelines to calculate the pre-coding matrix. As for the codebook based pre-coding method, you need to directly elect the receiving end of a matrix from a codebook as the pre-coding matrix, then the index matrix can be fed back to the 
receiver ${ }^{[4]}$. Fig.2 shows the calculation scheme of precoding matrix.

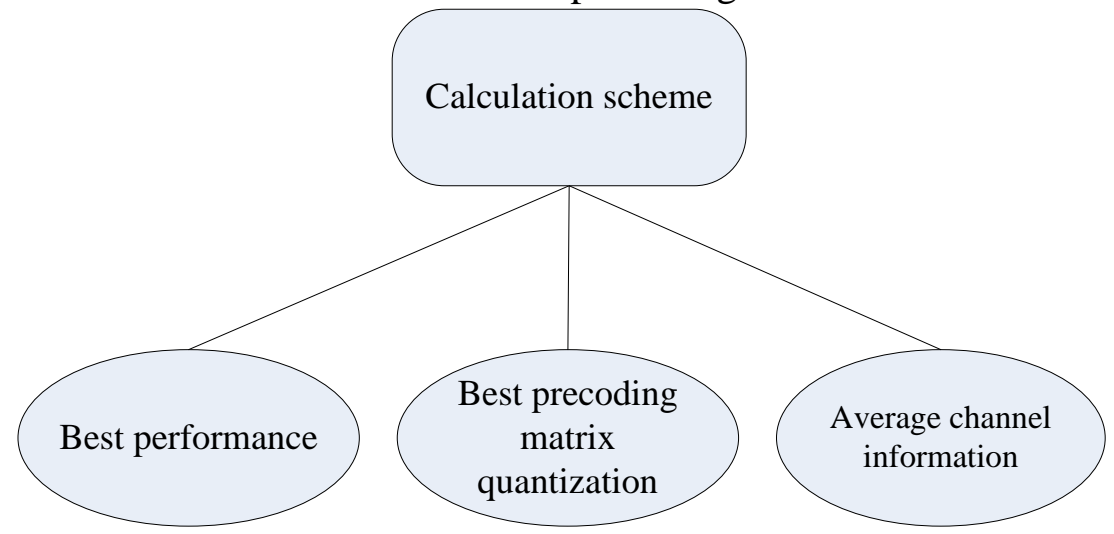

Fig. 2 .The calculation scheme of precoding matrix

In the case of codebook based pre-coding matrix only in a limited selection matrix, common choice can be divided into two categories: one is based on performance indicators selection, one is based on quantitative selection method. Select the method based on performance indicators refer directly to the codebook precoding matrix is selected when the corresponding performance index formula to calculate the weight, and then select the best performance of a matrix as a pre-coding matrix. Wherein the optimal pre-coding matrix can be calculated in accordance with the selection method based on the performance criteria. LTE precoding matrix information required between the transmitter and the receiver end of the transmission, which will take up a lot of valuable spectrum resources. Considering between OFDM symbol frequency correlation and temporal correlation, we can use the same pre-coding scheme on the frequency and time of a continuous OFDM symbols, thereby reducing the complexity of computing systems and feedback information.

\section{The LTE Key Technologies}

LTE system relative to the UMTS system, a revolutionary improvement in terms of multiple access technology, while in some other technical fields has been improved, such as the multi-antenna technology, adaptive technology. It also introduces some overall performance of innovative technologies for optimizing the system, such as cell interference suppression technology. The summary will be introduced for the LTE system in several key technologies ${ }^{[5]}$.

Orthogonal Frequency Division Multiplexing basic idea is that band is divided into a plurality of sub-parallel transmission of data, high-speed data stream into a plurality of parallel low-speed data stream, then modulated to each channel transmission subcarriers. The receiver channel measurements obtained for each subcarrier channel response information or SNR (Signal to Noise Ratio, SNR) estimation, thus use these channel state information, combined with a hybrid automatic repeat request technology to further fine-tune the throughput of the link. Adaptive modulation and coding is a link adaptation technique, adaptive coding modulation is essentially maintaining bit error rate (Bit Error Ratio, BER) premise constant, by adjusting the transmit power, selecting a modulation mode, or by selecting the modulation order to adapt to the dynamic changes of the SNR. AMC need to control the three key parameters: transmit power, modulation order and channel coding rate.

MIMO technology is the increased use of transport channel at the transmitter and receiver using multiple transmit and receive antennas, the use of space between the transceiver channel to improve data rate, reduce the bit error rate, so as to achieve the purpose of improving the quality of the wireless signal transmission. Since each transmit antenna signal transmitted simultaneously occupy the same frequency band, it is possible to improve the system have doubled capacity and spectral efficiency. MIMO technology includes transmit diversity and spatial multiplexing technology. Wherein transmission diversity is the signal on a different transmission antennas contain the same information, the use of space channels weak correlation, combined with time-selective frequencies, to improve the reliability of signal transmission. Spatial multiplexing refers to send different 
information on different transmit antennas using spatial channels weak correlation, thereby increasing the peak data transfer rate of remonstrance. Fig.3 shows the principle of multiple input and output.

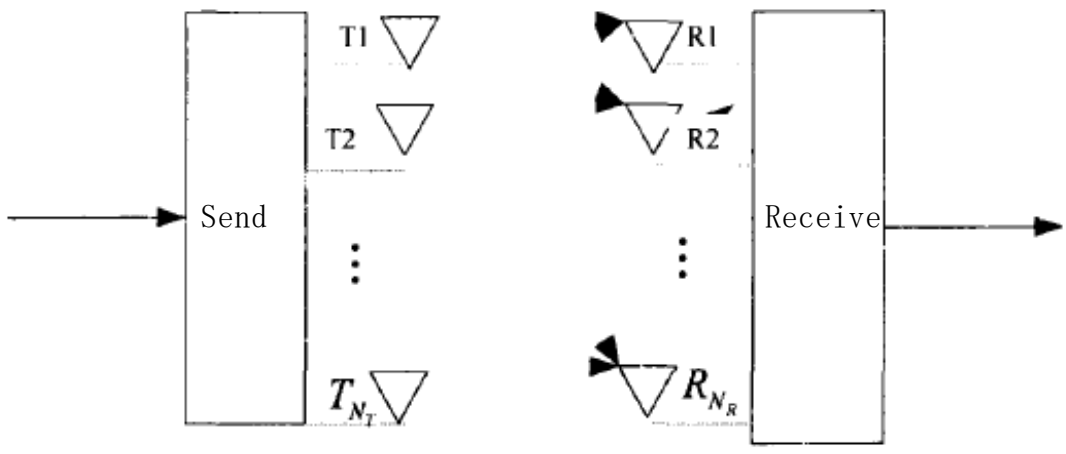

Fig. 3 .The principle of multiple input and multiple output

\section{Conclusions}

Precoding is one of the most effective approaches taken by the LTE standard to combating the rank-deficiency problem. In order to design a practical system, steps must be taken to reduce the probability of channel matrices with reduced ranks. This paper elaborated on the nature of channel-matrix rank deficiencies, introduced a pre-coding formulation and different types of pre-coding used in the LTE standard. Then the paper showed the design of LTE precoding and calculation scheme of precoding matrix. The article suggests that the optimal precoder can be determined by exploiting the singular-value decomposition of the channel matrix. Precoding performs linear transformations on the data of each substream to improve the overall receiver performance.

\section{Acknowledgement}

This work is supported by Scientific and Technological Research Program of Chongqing Municipal Education Commission(Grant No. KJ132206).

\section{References}

[1] S Feng, C Wen, L Han wen, Minimum MSE Based MIMO-OFDM Precoding Spatial Multiplexing Systems with Limited Feedback [C].Global Telecommunications Conference, 2007, 3057-3062.

[2] Sadek M, Tarighat A, Sayed A H, A Leakage-Based Precoding Scheme for Downlink Multi-User MIMO Channels [J]. Wireless Communications, 2007, 6(5):1711.

[3] V Stankovic, M Haardt, Generalized Design of Multi-User MIMO Precoding Matrices [J].Wireless Communications, IEEE Transactions, 2008, 7(3):953-961.

[4] Xiaojia Lu, Jin Han, Juntti M. Closed loop transmission for LTE system downlink [C]. European Wireless Conference 2009: 314-318.

[5] Schwarz S, Wrulich M, Rupp M. Mutual information based calculation of the Precoding Matrix Indicator for 3GPP UMTS/LTE[C]. Smart Antennas (WSA), 2010 International ITG Workshop. 2010: 52-58. 